\title{
A governação em SI: o caso da gestão das convenções e acordos de saúde do Algarve
}

\author{
Jorge Lami ${ }^{1}$, Silvia Fernandes ${ }^{2}$, Eric Vaz ${ }^{3}$ \\ Jorge.lami.leal@gmail.com, sfernan@ualg.pt, noronhavaz@gmail.com \\ ${ }^{1}$ Administração Regional de Saúde do Algarve, Largo de S. Pedro No ${ }^{15}, 8000-145$, Faro, Portugal \\ ${ }^{2}$ Faculdade de Economia, Universidade do Algarve, Campus Gambelas, 8005-139, Faro, Portugal \\ ${ }^{3}$ Universidade de Ryerson, 350 Victoria Street, Ontario M5B 2K3, Toronto, Canadá
}

DOI: 10.17013/risti.15.69-81

\begin{abstract}
Resumo: O presente trabalho é produto da investigação realizada numa instituição pública - Administração Regional de Saúde do Algarve (ARS Algarve) - a uma das áreas que tutela: as convenções e acordos de Meios Complementares de Diagnóstico e Terapêutica. Sem um sistema de informação que permita reter informação, e algum conhecimento tácito detido pelos seus profissionais, a gestão operacional desta área carece de eficiência. Isto levanta a necessidade de desenvolver um sistema de informação que cubra esta lacuna e sirva de suporte à prestação de informação útil ao utente. Foram estudados sistemas semelhantes, entrevistados especialistas do setor e realizados inquéritos a fim de desenvolver uma solução final ajustada às necessidades concretas dos utilizadores. Esta foi a grande preocupação do projeto, focando o estudo nos problemas, necessidades e aspirações daqueles que lidam com esta área.
\end{abstract}

Palavras-chave: inovação, empreendedorismo, gestão do conhecimento, gestão de projetos, convenções de saúde públicas.

\section{Governance in IS: the case of the management of conventions and health agreements of the Algarve}

\begin{abstract}
This work results from a research conducted in a public institution - the Regional Health Administration of Algarve (ARS Algarve) - on one of the areas it manages: conventions and agreements on Complementary Means of Diagnosis and Therapy. Without an information system that retains information, and some tacit knowledge held by its professionals, the operational management in this area lacks efficiency. This raises the need to develop an information system to cover this gap and serve to support the provision of useful information to users. Similar systems were studied, industry experts interviewed and surveys conducted in order to develop a final solution tailored to users' specific needs. This was the major concern of the project, focusing the study on the problems, needs and aspirations of those who deal with this area.
\end{abstract}

Keywords: innovation, entrepreneurship, knowledge management, project management, public health conventions. 


\section{Introdução}

O intra-empreendedorismo e a aplicação à área da saúde, a que o primeiro autor se encontra profissionalmente ligado (Administração Regional de Saúde do Algarve- ARS Algarve), estiveram na base do presente estudo. No departamento onde desempenhou funções na data da elaboração do estudo, onde é realizada a gestão das convenções e acordos de saúde de Meios Complementares de Diagnóstico e Terapêutica (MCDT), carecia de eficiência. A opção incidiu em estudar o problema e criar um sistema de informação (SI), inexistente na instituição, projeto este bem acolhido pelos decisores da organização. Os serviços de saúde requerem uma grande transformação no sentido de se obter maior interoperabilidade, eficiência e qualidade dos mesmos (Fernandes, 2010; Freixo \& Rocha, 2014). A necessidade de mudança conjugada com a dependência das organizações em relação aos SI requerem uma criteriosa seleção de metodologias. A falta de uma boa gestão da mudança é uma das principais causas do insucesso dos SI em saúde (Teixeira \& Rocha, 2008).

Para sustentar um projeto desta natureza (Meynhardt \& Diefenbach, 2012), foi necessário investigar a gestão de projetos e estudar outros SI para produzir um suporte adequado e ajustado às necessidades dos utilizadores. Não apenas as consideradas pelos investigadores, mas também as necessidades reais dos futuros utilizadores e daqueles que trabalham no seu dia-a-dia com SI de saúde. Assim, tornou-se necessário auscultar e inquirir especialistas e operacionais da área. Desenvolver um produto deste tipo na administração pública, com as atuais limitações financeiras e de recursos humanos, implicou que o impacto financeiro fosse minimizado desde a elaboração da proposta. Isso requereu constantes adaptações na implementação, em especial na componente de programação do software e parametrização da base tecnológica.

O presente artigo encontra-se estruturado em seis secções: a primeira introduz o tema. A segunda contextualiza o problema e apresenta os objetivos gerais do sistema proposto. A secção seguinte desenvolve a metodologia seguida, que incluiu entrevistas a especialistas e comparação com outros sistemas. Os resultados obtidos são discutidos na quarta secção, indicando os principais aspetos a incorporar no desenho do sistema. A secção seguinte descreve a solução em termos de organização funcional, desenvolvimento e desafios. Segue-se a conclusão que enfatiza as principais vertentes de atuação atuais e futuras.

\section{Contextualização do Problema}

A região do Algarve não dispõe de SI ou aplicação informática que permita gerir as convenções e acordos de âmbito regional de: análises clínicas e patologia clínica, anatomia patológica, cardiologia, medicina nuclear, eletroencefalografia, endoscopia gastrenterológica, medicina física e reabilitação, otorrinolaringologia, pneumologia, imunoalergologia, urologia, neurofisiologia, radiologia, especialidades médicocirúrgicas, psicologia e diálise.

Ficam patentes as necessidades de aumentar a qualidade, a consistência e a disponibilidade da informação gerada nesta área, tanto para os quadros afetos à sua gestão como para prestadores, prescritores e utentes, nomeadamente que tornasse mais fácil a identificação dos exames convencionados, em que locais e entidades. Os tempos de resposta e a qualidade do serviço são fundamentais nos SI em saúde (Laureano et al., 2014; Freixo \& Rocha, 2014). 


\subsection{Objetivos gerais}

São objetivos gerais do presente projeto:

- Gerir de uma forma eficiente as convenções de MCDT.

- Deter um repositório de informação geral: contratos, serviços disponíveis, licenciamentos, acordos, convenções, horários e moradas dos estabelecimentos.

- Deter uma aplicação que permita georreferenciar os recursos de saúde convencionados e os principais recursos do SNS no Algarve.

- Servir de meio facilitador da transmissão de informação para outras entidades (ARS Algarve, Administração Central do Sistema de Saúde, Entidade Reguladora da Saúde, entidades convencionadas).

- Aumentar a acessibilidade à informação específica, nesta área das convenções e acordos, nomeadamente a utentes e prescritores.

- Promover o intra-empreendedorismo e a capacidade de internalizar o desenvolvimento de uma solução informática, que em regra seria adquirida por concurso a entidades privadas.

Este projeto (SinC) pretende colmatar os problemas especificados e assim identificar, caracterizar, administrar e normalizar informação relevante de contratos, acordos e convenções existentes na região do Algarve. E permitir a sua publicação online para que fique acessível a utentes, prescritores, prestadores e quadros afetos (Departamento de Contratualização - Convenções e Departamento de Saúde Pública e Planeamento - Licenciamentos) e assim gerir de forma mais eficiente este processo complexo e multiparticipado.

A obtenção da denominação e posição georreferenciada das entidades convencionadas é hoje possível através do Portal da Saúde ${ }^{1}$, mas com informação muito reduzida e estática. Um objetivo deste projeto aplicado reside em fornecer ao utente informação específica e adequada, nomeadamente que exames e procedimentos estão disponíveis nas entidades convencionadas, ou numa determinada área geográfica, permitindo não só conhecer genericamente onde estão os convencionados, como limitar a sua pesquisa aos que disponibilizam os exames prescritos.

O projeto incidiu na investigação de vários modelos (benchmarking) e na apresentação de uma solução técnica (SI) que pudesse ser produzida internamente na ARS Algarve, racionalizando meios públicos e rentabilizando quadros qualificados. Este projeto é inovador na região e no país, suprindo necessidades atuais neste âmbito e ao mesmo tempo serve como exemplo de boas-práticas de intra-empreendedorismo na Administração Pública, essencial ao desenvolvimento do serviço público.

\section{Enquadramento e Metodologia}

A ARS Algarve aprovou a afetação dos recursos internos, nomeadamente na área de programação e engenharia de sistemas e comunicação, necessários ao desenvolvimento da solução. Foram aplicados questionários, numa adaptação do método Delphi, junto de

1 http://www.portaldasaude.pt/portal/servicos/prestadoresv2/pesquisa. 
diversos especialistas, dirigentes e profissionais de saúde no sentido de validar algumas opções específicas e desenvolvimentos futuros a propor.

Foi também usado o método de estudo de caso, com a análise de documentação de outros SI em uso no SNS, e realização de entrevistas exploratórias a profissionais encarregues da gestão de convenções, nomeadamente para aferir as necessidades existentes. Seguiu-se um inquérito a utilizadores para estudar os principais problemas dos SI (benchmarking) assim como suas vantagens e valor acrescentado para a área em estudo.

Foi desenvolvida uma análise funcional, testada por um painel de especialistas e gestores de convenções em sessões de brainstorming, para validar e adequar a proposta às necessidades reais. Na fase de prototipagem está programada uma sessão de design thinking com operacionais do setor das convenções e futuros utilizadores da aplicação, para testar a solução em desenvolvimento, nomeadamente em termos de ergonomia, e programar ajustes finais para obter um produto adequado às suas expetativas de utilização (Stewart \& Fortune, 1995).

Foi realizado um levantamento dos dados sobre convenções, sendo codificados e incorporados numa base de dados para facilitar a fase de testes do produto e permitir a transferência para a aplicação, por via eletrónica em vez de carregamento manual. Considerando que o desenvolvimento do projeto aplicado foi acolhido pela direção superior da organização, não foi necessário realizar um estudo de mercado nem foi considerado relevante assumir uma preocupação estatística (definição da amostra) pois o objetivo não seria estimar, mas testar o conceito e orientar a construção do SI.

\subsection{Inquérito Delphi}

Segundo Hasson et al. (2000), o método Delphi é uma técnica de facilitação de grupos, através de um processo de múltiplos estágios iterativos, especialmente útil para transformar a opinião geral num consenso de grupo. Esta técnica pode ser utilizada na investigação de projetos de SI (Keil et al., 1998) para identificar e categorizar as questões chave da solução.

O método Delphi foi adaptado a um inquérito online, onde especialistas da área da saúde, convidados para esse efeito, foram sucessivamente categorizando opções, re-submetidas até à obtenção da lista final que reunia consenso alargado. A opção pela aplicação deste método residiu na possibilidade de testar alternativas e melhorar o esboço inicial da arquitetura a utilizar e validar opções de desenvolvimento futuras, através da opinião de um leque variado de profissionais da área. Foram convidados médicos, enfermeiros, operacionais e especialistas da gestão de convenções, dirigentes e quadros superiores de diversas instituições do Ministério da Saúde.

Foram realizados inquéritos a 16 profissionais, $75 \%$ dos quais em funções no setor público (81\% com formação superior). A média de idades dos inquiridos é de 41 anos e $75 \%$ são do sexo feminino. Em termos de distribuição por tipo de entidade, 25\% desempenha funções num ACeS (Agrupamento de Centros de Saúde), 50\% numa ARS e 25\% numa entidade convencionada. Este inquérito permitiu testar e recolher informação sobre várias propostas, aplicando várias rondas até obter as mais consensuais. 


\subsection{Entrevistas}

Foram realizadas entrevistas semiestruturadas, de modo informal, que permitiram o enquadramento desta área das convenções, aferir necessidades específicas e percecionar atuais fragilidades e ineficiências, mas também pontos fortes e expetativas, nomeadamente dos operacionais da gestão em relação à solução final a apresentar. As entrevistas foram realizadas a vários profissionais, nomeadamente nas áreas da economia da saúde e da gestão de convenções, dos sistemas de informação e engenharia de sistemas. Foi desenhado um guião para auxiliar as entrevistas, ainda que as respostas não tenham sido codificadas. $\mathrm{O}$ objetivo foi obter um enquadramento geral e algumas pistas sobre as aspirações daqueles que foram envolvidos no projeto, para que a solução a desenvolver fosse ajustada às necessidades.

\subsection{Estudo de caso}

Carmo \& Ferreira (1998) enquadram o estudo de caso como uma abordagem empírica que investiga um fenómeno em contexto real. O estudo de caso foi suportado por duas técnicas complementares: 1) a investigação de dois SI em uso, nomeadamente ao nível dos manuais e literatura específica disponível, seguindo-se um teste às aplicações; e 2) um questionário online, essencialmente com o objetivo de gerar informação que orientasse o desenvolvimento da solução final.

Foram convidados a participar neste estudo de caso profissionais ligados às entidades convencionadas do SIGIC (Sistema Integrado de Gestão de Inscritos para Cirurgia) e utilizadores do SISO (Sistema de Informação para a Saúde Oral), bem como de instituições públicas como hospitais, outras regiões de saúde e serviços centrais que tutelam a área. Isto permitiu recolher informação no âmbito do conhecimento tácito detido pelos inquiridos, resultante de sua experiência quotidiana. O inquérito online foi realizado através do Google Forms e os dados foram trabalhados em SPSS e Excel.

Tabela 1 - SI em uso no Departamento de Contratualização

\begin{tabular}{lll}
\hline Sigla & Designação do SI & Gere convenções? \\
\hline SIGLIC & $\begin{array}{l}\text { Sistema informático de gestão da lista de } \\
\text { inscritos para cirurgia }\end{array}$ & Sim, na área cirúrgica \\
\hline SISO & Sistema de informação para a saúde oral & Sim, na área da saúde oral \\
\hline SICA & $\begin{array}{l}\text { Sistema de informação de suporte à } \\
\text { contratualização e acompanhamento }\end{array}$ & $\begin{array}{l}\text { Não, apoio à contratualização com os } \\
\text { ACeS }\end{array}$ \\
\hline RNU & Registo nacional de utentes & $\begin{array}{l}\text { Não, registo de utentes inscritos nos } \\
\text { centros de saúde }\end{array}$ \\
\hline SONHO & Sistema integrado de informação hospitalar & $\begin{array}{l}\text { Não, registo de utentes inscritos nos } \\
\text { hospitais }\end{array}$ \\
\hline & Sistema integrado de apoio à decisão & $\begin{array}{l}\text { Não, apoio ao acompanhamento do Centro } \\
\text { de Medicina Física e Reabilitação do Sul }\end{array}$
\end{tabular}


Em média, os inquiridos trabalham com SI em saúde há 7 anos, 5 dos quais no sistema em estudo e trabalham na área da saúde há 11 anos, 10 dos quais na instituição atual. Foram inquiridos profissionais que a nível local, regional e central utilizam aqueles dois SI, obtendo-se informação útil e procurando evitar enviesamentos. O Departamento de Contratualização (DC) trabalha com um conjunto de aplicações nas áreas que desenvolve, descritos na tabela seguinte.

Uma vez que dois dos SI usados são na área da gestão de convenções (SIGLIC e SISO), a opção recaiu no seu estudo mais aprofundado.

\section{Discussão de Resultados}

No contexto do estudo de caso (investigação de dois SI em uso), as questões foram categorizadas em função de: (i) caracterização geral do inquirido; (ii) adequação operacional do SI em estudo; (iii) comunicação e atendimento; (iv) problemas frequentes; (v) integração e formação no SI em estudo; (vi) adequação e robustez; e (vii) desenvolvimentos. Segue-se a apresentação de alguns dos principais outputs, obtidos com o software estatístico SPSS (Tabelas 2, 3 e 4). Na tabela 4, as médias devem ser interpretadas dentro da escala: "Nada adequado"=1; "Pouco adequado"=2; "Adequado"=3; e "Muito adequado"=4.

Tabela 2 - SI utilizados pelos inquiridos

\begin{tabular}{lccc}
\hline SI & Frequência & $\begin{array}{c}\text { Percentagem } \\
\text { válida }\end{array}$ & $\begin{array}{c}\text { Percentagem } \\
\text { acumulada }\end{array}$ \\
\hline SIGLIC & 18 & 64,3 & 64,3 \\
\hline SISO & 10 & 35,7 & 100 \\
\hline Total & 28 & 100 & \\
\hline
\end{tabular}

Tabela 3 - Gestão das convenções

\begin{tabular}{llll}
\hline $\begin{array}{l}\text { O SI melhora a gestão das } \\
\text { convenções? }\end{array}$ & Frequência & $\begin{array}{l}\text { Percentagem } \\
\text { válida }\end{array}$ & $\begin{array}{l}\text { Percentagem } \\
\text { acumulada }\end{array}$ \\
\hline Discordo & 1 & 3,6 & 3,6 \\
\hline Indiferente & 8 & 28,6 & 32,1 \\
\hline Concordo & 10 & 35,7 & 67,9 \\
\hline Concordo completamente & 9 & 32,1 & 100 \\
\hline Total & 28 & 100 & \\
\hline
\end{tabular}


Tabela 4 - Características com maior nível de concordância

\begin{tabular}{ll}
\hline Característica & Média \\
\hline O SI permitiu melhorar a prestação da área específica em que se insere? & 4,18 \\
\hline OSI melhora a gestão das convençôes? & 3,96 \\
\hline O SI melhorou a capacidade de tomar decisões de gestão? & 3,93 \\
\hline O SI melhora a comunicação com as entidades convencionadas? & 3,89 \\
\hline O SI permite reduzir custos com pessoal? & 3,89 \\
\hline O SI melhorou a capacidade de tomar decisões operacionais? & 3,75 \\
\hline Em termos de poupança de tempo e racionalização de outros recursos? & 2,89 \\
\hline Em termos de resposta às suas necessidades de informação? & 2,86 \\
\hline Em termos ergonómicos e de utilização física? & 2,79 \\
\hline Dificuldade em extrair informação útil & 0,57 \\
\hline Problemas de instabilidade de ligação & 0,46 \\
\hline Problemas de instabilidade do sistema & 0,43 \\
\hline Interface & 0,29 \\
\hline Menus complicados & 0,21 \\
\hline Ergonomia inadequada & 0,11 \\
\hline Perda de informação & 0,11 \\
\hline Informação errada & 0,11 \\
\hline
\end{tabular}

As médias da tabela 4 permitem ordenar as características mais pontuadas pelos inquiridos em relação aos dois SI estudados (SIGLIC e SISO), em função do seu nível de concordância.

Este benchmarking permitiu conhecer melhor dois SI semelhantes ao sistema em questão (SinC), fornecendo importantes informações para o seu desenvolvimento, formação e subsequente apoio, colmatando falhas de conceção não identificadas. No seguimento destes resultados, e também de reuniões e sessões de brainstorming no âmbito do projeto, os principais aspetos a incorporar no desenho do SinC são:

- Permitir a comunicação entre as diversas entidades, automatizando procedimentos que apoiem a gestão dos processos a desenvolver, de forma a reduzir o tempo de trabalho, gerando eficiências com impacto na redução do custo com pessoal podendo dedicar-se a outras tarefas.

- $\quad$ Dispor de opções para obter informação operacional, mas também dashboards que permitam monitorizar a situação atual das convenções, sem necessidade de realizar consultas/relatórios ad hoc.

- Possibilidade de parametrizar relatórios específicos e dispor de alguns relatórios padrão, em função das necessidades atuais, com as datas em que as convenções expiram e avisos/alertas para facilitar a gestão dos prazos.

- Dispor da possibilidade de carregar documentos eletrónicos como os pactos sociais, licenças de funcionamento e outros documentos de controlo, para evitar consumos de tempo em arquivo ou necessidade de gerar mais papel. 
- Mapa de contactos que facilite o acesso a informação resumo das entidades convencionadas e dos exames que convencionam.

- Simplicidade nos menus e ergonomia de utilização (acesso à informação com o mínimo de passos).

- Sistema fiável, sem requerer demasiados recursos e memória do computador ou da rede.

- Disponibilidade permanente do helpdesk com capacidade para desenvolver alterações decorrentes de mudanças legislativas ou operacionais.

- Estudar o desenvolvimento futuro da aplicação para incluir suporte wireless, nomeadamente para uso em tablets.

\subsection{Fatores de sucesso}

Em suma, os principais fatores de sucesso do sistema resumem-se na tabela 5 .

O ambiente interno e externo não são estáveis ao longo do tempo, pelo que são de difícil previsão. Os fatores da Tabela 5 foram considerados no desenvolvimento do projeto, em que uma incorreta avaliação destes pode comprometer a solução a apresentar. Estes fatores foram também relevantes na elaboração do plano de comunicação.

A metodologia utilizada permitiu suportar um conjunto de decisões orgânicas, nomeadamente no modelo de interação tecnológica abaixo explicitado e sobretudo na fase conceptual e de arquitectura, clarificando a definição de menus e outros elementos a incorporar no software. E o estudo de caso, ao analisar SI desenvolvidos para outras áreas, deu contributos importantes para este projeto, evitando erros de conceção ou insistência em características que os potenciais utilizadores não consideram relevantes ou necessários. Isto encurta a curva de experiência (tentativa, erro, correção) da equipa de desenvolvimento nos aspetos mais técnicos no domínio das convenções. As entrevistas e a aplicação do método Delphi permitiram concentrar os outputs em necessidades de informação pertinentes e envolver os profissionais de vários níveis (nacional, regional e local) e com várias funções na divulgação e dinâmica do projeto, o que permitirá aumentar o grau de reconhecimento e aceitação.

\section{A Solução - SinC}

Após as secções anteriores, que permitiram conhecer melhor a organização, enquadrar o problema e auscultar outros SI, apresenta-se a seguir o enquadramento específico, conceção e desenho da solução (SinC).

O SinC é uma proposta de solução integrada (Figura 1), dividida em dois módulos com características distintas, quer em relação ao público a que se dirige quer ao nível do problema a resolver: 1) gestão das convenções (GC); e 2) website para comunicar com os utentes em tempo real (AS360), que permite distribuir informação organizada e categorizada obtida no GC (nomeadamente georreferenciada) dos recursos de saúde disponíveis no Algarve, públicos e convencionados, para além da capacidade de receber inputs dos utilizadores.

Inserindo-se no ambiente web 2.o (O’Reilly \& Battelle, 2009), pretende-se centrar esta solução no utilizador, não apenas profissional (interno ou externo) mas sobretudo no 
Tabela 5 - Fatores críticos de sucesso

\begin{tabular}{ll}
\hline Fator & Objetivo \\
\hline Divulgação & $\begin{array}{l}\text { Compromisso dos profissionais de saúde e de contato em divulgar e utilizar } \\
\text { o produto, evitando os atuais contactos telefónicos à equipa operacional das } \\
\text { convenções, nomeadamente para obter a localização dos convencionados onde são } \\
\text { realizados determinados exames específicos }\end{array}$ \\
\hline $\begin{array}{l}\text { Adequação } \\
\text { técnica }\end{array}$ & Estabilidade da ligação ao servidor; fiabilidade e integração do SI \\
\hline $\begin{array}{l}\text { Recursos } \\
\text { humanos }\end{array}$ & $\begin{array}{l}\text { Estabilidade na equipa de gestão das convenções; equipa técnica com os } \\
\text { do tempo e com capacidade crítica para pensar a médio-longo prazo, no sentido de } \\
\text { oferecer na fase de desenvolvimento informação abrangente para que o produto } \\
\text { tenha a maior longevidade possível }\end{array}$ \\
\hline & $\begin{array}{l}\text { Perceção do valor associado à utilização deste produto, nomeadamente em termos de } \\
\text { tempo de resposta, menor custo, flexibilidade, disponibilidade, credibilidade e maior } \\
\text { amplitude de conteúdos úteis da resposta, como alternativa ao serviço por telefone } \\
\text { ou presencial; perceber exatamente que necessidades principais e assessórias ao nível } \\
\text { dos pedidos devem ser satisfeitas com o SI }\end{array}$ \\
\hline
\end{tabular}

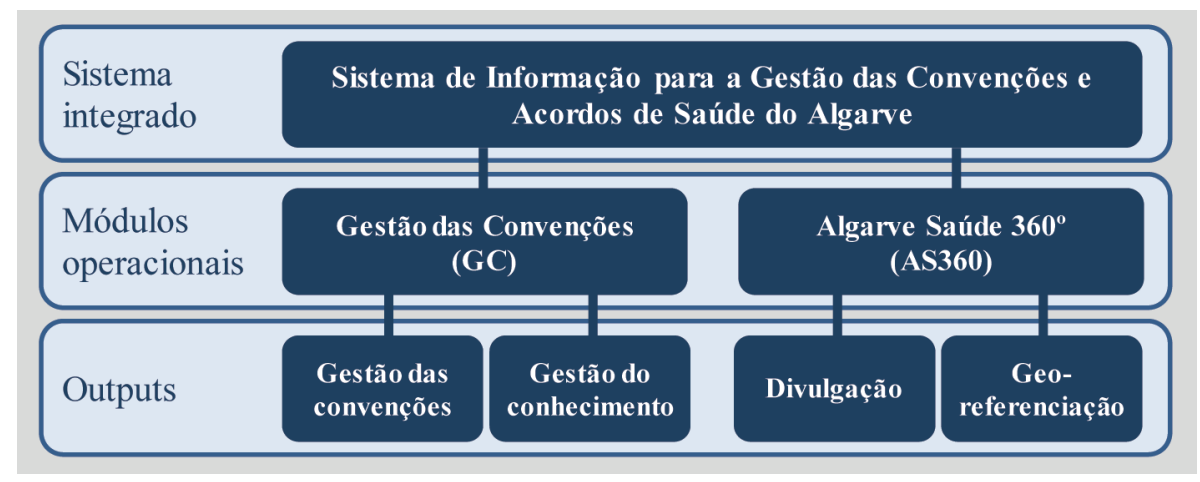

Figura 1 - Organização do SinC

utente que pode comunicar e interagir com a ARS Algarve por este meio. Não apenas para obter a informação que necessita na área das convenções e acordos, mas também para reclamar, expor, elogiar ou recomendar nas redes sociais as suas experiências com entidades convencionadas, participando no esforço de comunicação deste sistema.

\subsection{Organização funcional}

Propõem-se dois níveis complementares para o desenvolvimento da solução: nível de projeto e nível operacional. A equipa de projeto é incumbida de desenvolver o produto, aplicar o plano de comunicação e avaliar a implementação operacional, emitindo as 
recomendações e desenvolvendo os ajustes necessários. Esta equipa será extinta 6 meses após a entrada em funcionamento pleno do SI, ainda que os especialistas de informática se mantenham como equipa de manutenção e apoio sob a responsabilidade funcional do Núcleo de Sistemas de Informação e Comunicação da ARS Algarve.

A equipa operacional das convenções de MCDT, integrada no Departamento de Contratualização, exerce as funções técnicas e administrativas da gestão das convenções. Constitui um elemento essencial ao desenvolvimento do SI e foi envolvida desde a fase de investigação da solução. Na elaboração do projeto, a mesma foi constantemente envolvida, inquirida, incluída nos testes de conceito e na prototipagem, porque será esta que diariamente utilizará o SinC.

\subsection{Conceção e desenvolvimento da solução}

O Ministério da Saúde desenvolveu uma VPN² encriptada - a Rede de Informação da Saúde (RIS) - que permite garantir a segurança e controlo de acessos, a confidencialidade dos dados de saúde, entre outros aspetos. Esta rede não permite o acesso geral, pelo que a aplicação SinC é desenvolvida com a Intranet. Esta foi a solução mais adequada para dar resposta às necessidades de acesso, integração e partilha da informação, nomeadamente devido à necessidade de ligação externa fornecendo a informação necessária ao website AS360 e permitindo que as entidades convencionadas se liguem à aplicação (sem necessidade de possuir um IP ${ }^{3}$ fixo).

\subsubsection{Ciclo de vida do produto e desafios}

Planear elementos que permitam evitar o declínio do produto é algo que deve ser incorporado no desenvolvimento. A introdução de melhorias e novas ferramentas (Código QR e Apps) permite manter a maturidade e adequação do produto com resposta sustentada para os problemas identificados. O controlo sobre o desenvolvimento, realizado por meios próprios e salvaguardando a propriedade e acesso ao código do programa, é relevante para atualizar o SI sempre que necessário.

As linguagens de programação utilizadas estão relacionadas com dois vetores: (i) necessidades técnicas face aos objetivos do produto final; e (ii) competência dos recursos humanos envolvidos na programação. Tais linguagens são HTML 4 (para a construção do site, por permitir a interpretação generalizada dos navegadores de internet); Javascript (linguagem de programação que aumenta a interatividade, usabilidade e nível de pormenores visuais das páginas); e $\mathrm{PHP}^{5}$ (pela rapidez de processamento, manutenção simples e exequível em qualquer plataforma).

O sistema de gestão de bases de dados utilizado foi o MySQL, por ser de utilização livre, interagir com PHP e pela rapidez com que realiza pesquisas nos dados. Para garantir maior segurança operacional, será utilizado o protocolo HTTPS ${ }^{6}$ que, através da encriptação da conexão de dados, incrementa a segurança das comunicações entre

2 Virtual Private Network.

3 Internet Protocol.

4 HyperText Markup Language.

5 HyperText PreProcessor.

6 HyperText Transfer Protocol Secure. 
clientes e servidor. A componente de referenciação geográfica do website utiliza o Google Maps como fornecedor e gestor de localizações. A opção prende-se com a facilidade de utilização, estabilidade da ligação e por não ter custos para o número de referências que serão utilizadas.

Conforme a figura 2, os utilizadores da ARS Algarve têm acesso ao GC e ao AS36o por via da RIS, onde está instalado o servidor que aloja o SinC. Os utilizadores externos, entidades convencionadas e população em geral, fazem o seu acesso via ligação protegida ao servidor (através da internet).

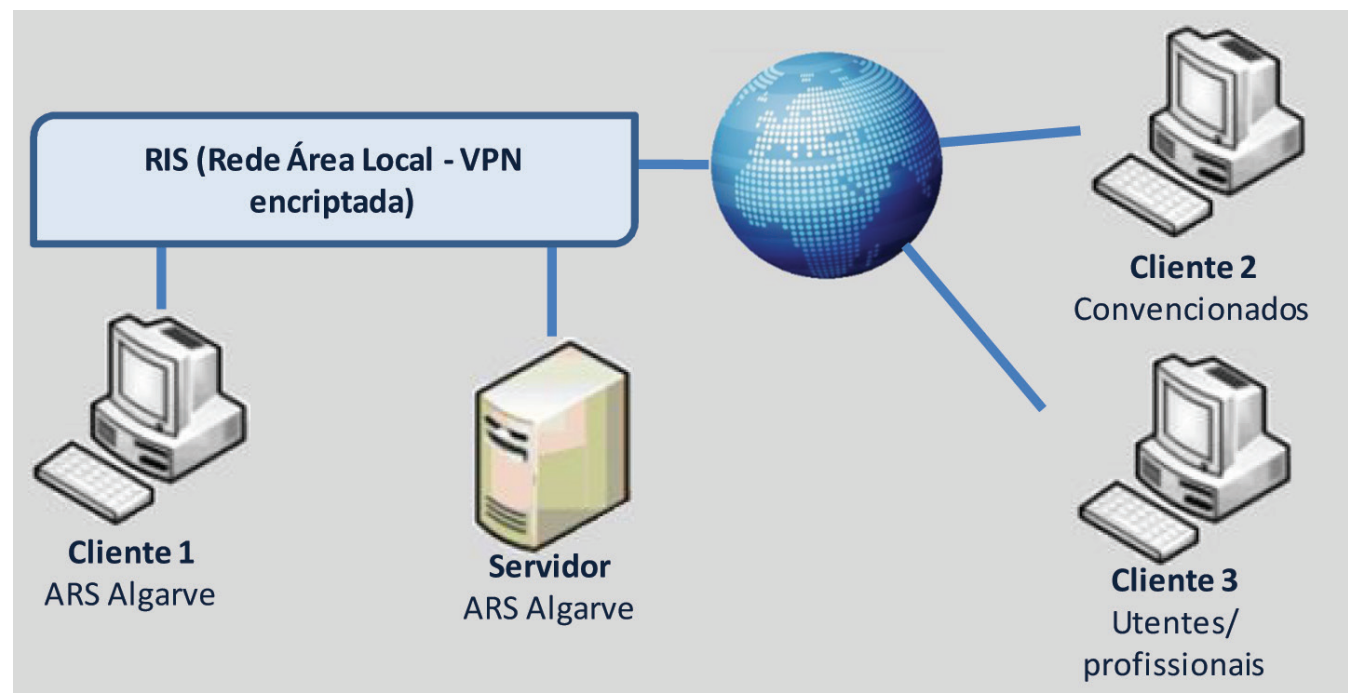

Figura 2 - Vista de estrutura do hardware

$\mathrm{Na}$ fase de prototipagem, serão convidados a participar elementos que lidam com aplicações de outras áreas, para obter um design adequado e arquitetura robusta. Depois dessa fase a programação é finalizada e o produto é colocado online.

O desenvolvimento e aplicação do projeto teve variados desafios, que resultaram das limitações de recursos humanos disponíveis e da necessidade de priorizar desenvolvimentos em SI clínicos, nomeadamente rastreios oncológicos e de cobrança de taxas moderadoras em postos de praia. Ocorreram ainda alterações estruturais, como a mudança de instalações da sede da ARS Algarve, que retirou durante vários meses os colaboradores da área de SI para o suporte das migrações, mudanças de servidores e restante plataforma informática, para além das configurações necessárias em mais de uma centena de postos de trabalho. Existiram também condicionantes que decorrem dos processos de contratualização com hospitais e cuidados de saúde primários no início do ano, retirando colaboradores essenciais à gestão deste projeto. 


\section{Conclusão}

Com o SI proposto é esperada a consolidação da informação sobre acordos e convenções, assim como a simplificação e disponibilidade de acesso, considerando que se encontra atualmente dispersa em suporte papel. Em suporte eletrónico permite codificar conhecimento útil e automatizar algumas funções, nomeadamente ao nível do controlo e gestão de processos das convenções. É criada a oportunidade de aproximar os convencionados e os utentes, promovendo a participação mais ativa destes últimos na obtenção da informação desejada. Promove também uma participação mais ativa dos profissionais de saúde e de apoio ao utente, na obtenção e prestação de informação aos seus públicos-alvo.

O maior desafio na implementação do sistema proposto, desenvolvido com utilização exclusiva de recursos próprios de uma instituição pública neste período de limitação de recursos (ANAO, 2009), prende-se com imprevistos que possam ocorrer noutras áreas e requeiram a desafetação de recursos deste projeto. Ao nível operacional ocorreram alterações nas prioridades dos sistemas de informação em desenvolvimento, o que se traduziu numa limitação dos recursos disponíveis para a equipa deste projeto.

A solução encontrada apresenta duas vertentes distintas, uma dirigida à gestão das convenções (GC) e outra para a divulgação georreferenciada das entidades de saúde convencionadas e respetivos exames (AS360):

- GC: é esperado um tempo de adaptação curto, uma vez que os operacionais foram envolvidos desde a arquitetura do sistema, contribuindo para que fosse adequado às suas necessidades e expetativas (ergonomia de utilização e organização interna). O maior esforço antecipado reside na divulgação junto das entidades convencionadas. No entanto, considerando que a entidade promotora é reguladora regional, não são esperados problemas na introdução e aceitação do sistema;

- AS360: esta componente é dirigida à faixa de utilizadores com conhecimentos e acesso à internet. É de notar que uma parte da população (mais idosa ou menos escolarizada), a quem são prescritos exames a realizar em entidades convencionadas, não tem acesso direto à internet. Assim, o contacto telefónico com os profissionais de saúde e assistentes técnicos será substancialmente melhorado. Como qualquer tecnologia, será necessário ganhar a confiança dos profissionais dos cuidados de saúde primários e garantir que a aplicação esteja sempre funcional e atualizada.

Este projeto foi dimensionado para dar resposta a dois problemas: (i) gerir as convenções de MCDT de forma eficiente e (ii) permitir que os utentes e profissionais de saúde obtenham informação sobre estas convenções e onde as mesmas estão disponíveis no Algarve. No entanto, outros elementos poderão ser alvo de desenvolvimento futuro tais como:

- Impressão das entidades que realizam os exames ou as consultas, na própria prescrição.

- Melhorar a comunicação da ARS Algarve com banners de informação sobre rastreios (utentes) e outros eventos de saúde (profissionais), entre outras 
informações úteis (informação sazonal sobre disponibilidade de equipas de saúde durante o período balnear e localização dos equipamentos mais próximos).

- Sistema de informação geográfica dos equipamentos e instalações de saúde do Algarve, com informações da área de saúde pública.

- Apps para acesso ao GC e ao AS36o por iOS e Android.

- Código QR nas prescrições para acesso à página dos locais de prestação das entidades convencionadas.

\section{Referências}

ANAO (2009). Innovation in the Public Sector: Enabling Better Performance, Driving New Directions. Report, Australian National Audit Office.

Carmo, H. \& Ferreira, M. (1998). Metodologia da Investigação: Guia para a autoaprendizagem. Lisboa: Universidade Aberta, ISBN: 972-674-231-5.

Fernandes, S. (2010). Diagnóstico do SI do INEM: Fragilidades e Mudanças em Curso. In Rocha, Á. (Ed.), Sistemas e Tecnologias de Informação em Saúde. Porto: Edições Universidade Fernando Pessoa, 63-73.

Freixo, J., \& Rocha, Á. (2014). Arquitetura de Informação de Suporte à Gestão da Qualidade em Unidades Hospitalares. RISTI - Revista Ibérica de Sistemas e Tecnologias de Informação, (14), 1-15. doi: http://doi.org/10.17013/risti.14.1-15

Hasson, F., Keeney, S. \& McKenna, H. (2000). Research guidelines for the Delphi survey technique, Journal of Advanced Nursing, 32(4), 1008-1015. doi: http://doi. org/10.1046/j.1365-2648.2000.to1-1-0156

Keil, M., Cule, P., Lyytinen, K. \& Schmidt, R. (1998). A framework for identifying software project risks, Communications of the ACM, 41(11), 76-83. doi: http://doi. org/10.1145/287831.287843

Laureano, R., Caetano, N. \& Cortez, P. (2014). Previsão de tempos de internamento num hospital português: Aplicação da metodologia CRISP-DM. RISTI - Revista Ibérica de Sistemas e Tecnologias de Informação, 13, 83-98. doi: http://dx.doi. org/10.4304/risti.13.83-98

Meynhardt, T. \& Diefenbach, F. (2012). What Drives Entrepreneurial Orientation in the Public Sector? Evidence from Germany's Federal Labor Agency, Journal of Public Administration Research and Theory, 22(4), 761-792. doi: http://dx.doi. org/10.1093/jopart/muso13

O'Reilly, T. \& Battelle, J. (2009). Web Squared: Web 2.o Five Years On, Special Report. Disponível em http://assets.en.oreilly.com (acedido em 14 de Julho de 2014).

Stewart, R. \& Fortune, J. (1995). Application of systems thinking to the identification, avoidance and prevention of risk, International Journal of Project Management, 13(5), 279-286. doi: http://dx.doi.org/10.1016/0263-7863(95)ooo24-K

Teixeira, P. \& Rocha, Á. (2008). Gestão da mudança de sistemas de informação em unidades de saúde: Proposta de investigação. RISTI - Revista Ibérica de Sistemas e Tecnologias de Informação, 1, 78-82. 